\title{
COSA ACCADE DI LÀ DALL'OCEANO? DIRITTO E LETTERATURA IN EUROPA ${ }^{1}$
}

\section{Paola MitTica ${ }^{2}$}

\begin{abstract}
RiassunTo: Obiettivo di questo contributo è delineare il quadro attuale degli studi di "Diritto e letteratura" da intendersi come una metodologia che riflette sull'interdisciplinarietà come via necessaria per elaborare un'osservazione complessa dei fatti dell'uomo e delle sue relazioni. Ma può divenire anche un movimento nell'orizzonte dell'impegno civico e politico, qualora alla cultura dell'interdisciplinarietà si coniughi quella dell'alterità e della responsabilità. Nel riassumerne le linee essenziali, l'esperienza europea sarà messa a confronto con quella statunitense per evidenziare la necessità di un recupero critico dell'identità culturale europea, da cui muovere in modo più costruttivo e originale all'evoluzione della prospettiva. Con particolare riferimento alla "rinascita" italiana degli studi di Diritto e letteratura dell'ultimo ventennio, saranno poi delineate alcune distinzioni di campo, utili a individuare tra i diversi versanti di Law and Humanities quelli che segnano l'ambito specifico di Diritto e letteratura, per tracciare, infine, all'interno di quest'ultimo, alcune direttrici di ricerca condivisibili interdisciplinariamente.
\end{abstract}

Parole-Chinve: Diritto e letteratura; Studi europei; Interdisciplinarietà; Transdisciplinarietà.

\section{BREVI RIFERIMENTI A UNA STORIA NOTA}

La storia più nota di Law and Literature comincia nelle Law School statunitensi, dove questi studi nascono, sin dai primi anni del secolo scorso,

\footnotetext{
${ }^{1}$ Il testo riprende in larga parte i contenuti di una relazione tenuta in occasione dell'edizione Piazza delle Lingue 2013 organizzata dall'Accademia della Crusca (Firenze) e comparsa con il titolo Diritto e letteratura. Disciplina, metodologia o movimento? (Mittica, 2014).

2 Postdottorato presso la Facoltà di Sociologia dell'Università di Urbino (Italia). Professore Associato di Filosofia e Sociologia del Diritto presso il Dipartimento di Giurisprudenza dell'Università degli Studi di Urbino. Coordinatrice Scientifica della Italian Society for Law and Literature (ISLL), presso il CIRSFID dell'Università degli Studi di Bologna. E-mail: maria.mittica@uniurb.it
} 
per la didattica. L'obiettivo è di coltivare la sensibilità degli operatori giuridici e politici attraverso l'analisi e la diffusione di opere letterarie - per lo più di tradizione anglosassone - in cui si rinvengono i valori giuridici fondamentali della cultura americana, ma nella storia più recente - a partire dagli anni '70 - Law and Literature si trasforma in uno dei movimenti di rivolta contro il formalismo nel solco dei Critical Legal Studies (CLS), proiettato verso una politica del diritto e dei diritti animata dalle istanze critiche del realismo giuridico (Minda, 1995; Sansone, 2001).

È proprio nel quadro di questa lotta che Diritto e letteratura matura nei suoi aspetti più complessi e interessanti, tornando all'attenzione dei critici del diritto in modo rinnovato e in un proficuo connubio con gli studi letterari. Il mutamento è così significativo che si finisce per stabilire convenzionalmente una "rinascita" degli studi di Law and Literature nel 1973, anno in cui viene pubblicato The Legal Imagination di James Boyd White (1973), divenuto ormai un testo icona a testimonianza che da qui in poi, per gli studiosi che vorranno accostarvisi, Law and Literature deve essere inteso come un movimento volto a rinnovare il discorso politico e giuridico ma a partire da una ricerca attenta alla realtà sociale e umana3 . La risposta alla "call to context" consiste nel ricondurre al contesto i "discorsi" e i numerosi studi interessati da quella "tessitura del senso" che si compie nel corso della vita quotidiana, nello specifico a opera della legge o della letteratura, ma in generale per mezzo di tutti i testi che incidono nella creazione e nella riproduzione dei significati che regolano le relazioni.

Ponendo come punto di partenza l'osservazione della vita come "vita del linguaggio" - stando alla lezione dello stesso White (1984) -, da questa prospettiva il concetto di letteratura abbraccia, dunque, molte più species testuali di quante siano individuate correntemente come letterarie, avvicinandosi maggiormente al campo delle scienze del testo che non a quello della letteratura, laddove anche il diritto viene osservato come uno tra i testi culturali aventi la funzione di fissare significati e costituire una

\footnotetext{
${ }^{3}$ Non a caso, James Boyd White è per sua formazione sia critico letterario che giurista. Quando cominciò a studiare diritto, ama ripetere, si stupì di trovarsi in un ambiente "critico" assolutamente familiare a quello da lui frequentato durante gli studi di letteratura (White, 2011).
} 
comunità nel linguaggio4. L'ulteriore esito è che l'interesse per il diritto coinvolga non più soltanto i giuristi, ma anche i letterati e in generale gli studiosi del testo 5 .

Certo, il posizionamento dell'assunto è forte e per certi aspetti anche spaesante, in quanto impone alle varie scienze coinvolte di riportare le relative specializzazioni a un presupposto comune e trasversale, che richiede un'effettiva mediazione interdisciplinare con uno sforzo di interpretazione, traduzione e ricombinazione di categorie e lessici non indifferente ${ }^{6}$. Tant'è che parte della ricerca di Law and Literature, pur sposando molte delle sollecitazioni della "rinascita", preferisce limitarsi a riflettere sui termini tradizionali del binomio diritto e letteratura, e piuttosto approfondirne le implicazioni al livello teorico e metodologico.

Inutile dire che tra questi due estremi lo sviluppo del campo è divenuto talmente ampio e variegato da rendere impossibile una ricostruzione esaustiva. Ci limitiamo pertanto a rilevare alcune evoluzioni che sembrano emergere con particolare rilievo.

Innanzi tutto, sostenuto dall'elaborazione di una nozione di testo che contempla anche l'oralità piuttosto che altre forme comunicative e artistiche, va segnalato lo sbocco delle istanze di White in un campo di ricerca che si è esteso dalla letteratura all'intero ambito delle humanities ${ }^{7}$. Si tratta di Law and Humanities in cui si inscrivono, accanto a Diritto e letteratura, anche Diritto e cinema, Diritto e musica, Diritto e arte (pittura, scultura, fotografia, video arte, ecc.), con l'invito a esplorare i

\footnotetext{
${ }^{4}$ White mette insieme testi di vario genere: epica (Iliade), storia (Tucidide), filosofia (Platone), romanzi (Swift, Austin, Johnson), filosofia della storia (Burke), testi costituzionali americani (diritto). L'esperimento è di tipo metodologico: non si tratta di analizzare soltanto per se stessi i vari testi, ma di interrogarli su quello che hanno da dirci circa il nostro modo di costruire significati comuni, contestualizzandoli al momento in cui il lettore legge.

${ }^{5}$ Vale la pena sottolineare che l'esperienza statunitense si pone sin dalle prime battute della "rinascita" in chiave interdisciplinare. Ne sono testimonianza sia i numerosi convegni e seminari e le opere collettanee che coinvolgono studiosi provenienti dalle diverse aree specialistiche (significativo in tal senso il primo numero della Cardozo Studies in Law and Literature, del 1989), sia la propensione di molti ricercatori ad assumere in prima persona - come White - una doppia competenza.

${ }^{6}$ In questa direzione, White (1987-1988) promuove un lavoro interdisciplinare che divenga capace di negoziare tra i vari linguaggi specialistici, per giungere a una mediazione linguistica (intesa anch'essa come "letteraria"), nell'ottica di una nozione di letteratura ampia al punto da identificare il linguaggio condiviso.

7 Sebbene, a rigore, la nozione di "letteratura" utilizzata da White potrebbe essere interpretata in maniera ancora più ampia, e superare quella di Humanities.
} 
nessi tra il diritto e le varie humanities, tenendo sullo sfondo anche l'istanza che la formazione giuridica, ma più propriamente un'autentica formazione liberal indirizzata ai comuni cittadini (tra i quali i giuristi che giocano un ruolo sociale tra i più significativi), non può prescindere da un'attenzione per la "cultura" che deve declinarsi consapevolmente come sensibilità verso la complessità della vita in comune per creare le condizione di un accostamento "simpatetico" all'Altro (Sarat et al., 2010).

Con un approccio per certi versi analogo, sulla scorta dell'idea che le comunità umane e politiche siano narrative e allo stesso tempo normative, si sviluppa Law as narrative, attraverso cui si osserva come "giuridica" tutta l'attività del raccontare, che ha per effetto la definizione di un ordine simbolico e comportamentale, e dunque anche il diritto come una pratica o un insieme di pratiche narrative (Mittica, 2010). Su questo versante, al celebre saggio di Robert Cover (1983) - in cui dalla messa in rapporto dei termini "nomos" e "narrazione" si sviluppa un'analisi critica del diritto e del modus operandi delle Corti federali degli Stati Uniti, che sfocia in un costituzionalismo impegnato nella progettualità della vita sociale -, si accosta il movimento del legal storytelling, con l'ambizione a far emergere le "voci escluse" dalla legge nel discorso pubblico, restituendo valore al patrimonio giuridico presente nelle prassi sociali e alle aspettative normative dei gruppi minoritari che non trovano cittadinanza ${ }^{8}$. Un ulteriore profilo di Law as narrative si coniuga, infine, con la dimensione identitaria dell'uomo sociale (politico e giuridico), il quale, tramite la tensione alla narrazione, osservata come caratteristica della mente umana, costruisce il proprio progetto di vita e lo condivide nel contesto comune ${ }^{9}$.

\footnotetext{
${ }^{8}$ Il clima effervescente di ricerca, sperimentazione e creatività in cui nascono questi studi è ben testimoniato da un simposio pionieristico sulla narrazione giuridica lanciato dalla Michigan Law Review nel 1988 su proposta di Richard Delgado, i cui esiti saranno pubblicati in un numero monografico della rivista intitolato Legal Storytelling (Michigan Law Review, v. 87, n. 8, p. 2069-2494, aug. 1989), oltre gli studi di Ewick e Silbey (1995) e di Silbey (2005).

${ }^{9} \mathrm{Si}$ ascrivono a questo approccio principalmente le tesi di Jerome Bruner (1991, 2000, 2002) che fonda un indirizzo di ricerca, la psicologia culturale, che ha assunto un significativo rilievo anche negli studi italiani di concerto con la sociologia narrativa.
} 
Quanto al versante metodologico, uno degli esiti più interessanti riguarda l'elaborazione di una critica letteraria del diritto. In un importante studio, Robert Weisberg e Guyora Binder (2000, p. 25) riassumono tutti i generi di critica letteraria del diritto, intendendo il diritto di volta in volta come "pratica interpretativa" (ermeneutica); come "pratica narrativa" (costruzione di storie); come "attività volta a persuadere, decidere, dialogare" (retorica); come "linguaggio" (che attraverso le lenti del decostruzionismo è osservato come una pratica che configura la superficie della realtà nascondendone il lato oscuro e violento). Tutte dimensioni queste, secondo gli autori, strettamente collegate e riconducibili a una più ampia prospettiva culturale, per cui il diritto va inteso principalmente come "pratica compositiva di un tipo di manufatto letterario", vale a dire come una rappresentazione della realtà - che assuma la forma di una lettura interpretativa, dell'elaborazione di una storia, di una performance o di un segno linguistico. In altre parole, la critica letteraria ritrarrebbe il diritto come un'arena in cui giocano tanto gli aspetti strutturali quanto quelli propriamente volontaristici (scelte e azioni degli attori sociali) dell'universo simbolico che ordina la vita in comune, espressi secondo le modalità e nelle forme del genere letterario. Per questa via, dunque, "attrezzi" della critica letteraria contribuirebbero al disvelamento di elementi particolaristici e spesso strumentali sottaciuti dietro la maschera del formalismo giuridico (Brooks, 2011).

Dall'altro lato, del "contenimento", per così dire, di Diritto e letteratura entro i confini più definiti del binomio è, invece, il filone che tradizionalmente accosta il diritto ai grandi classici della letteratura occidentale, ancorato sia all'approccio metodologico Law in literature, ovvero all'analisi della rappresentazione letteraria di tematiche che hanno rilievo per il diritto, sia all'idea che la formazione giuridica e civica ritenuta il principale sbocco di Law and Literature - possa trovare una profonda e più complessa visione della realtà dell'uomo soltanto nelle opere che hanno segnato la cultura occidentale (Posner, 2009). 
$\mathrm{Al}$ di là di precise obiezioni verso le istanze più innovative del movimento e di una certa diffidenza per un'interdisciplinarietà che non a torto si teme possa scivolare nel mero dilettantismo ${ }^{10}$, su questo versante si colloca anche la ricerca di quanti lavorano all'elaborazione di un'etica in grado di guidare un progetto politico ispirato da valori che supportino lo sviluppo umano, contrastando modelli di "crescita” basati principalmente sull'utilitarismo economico che si sono rivelati escludenti e deficitari. I lavori che più lo ricordano sono quelli di Martha C. Nussbaum (1995, 1997, 2001, 2004), la quale, sul terreno di un'ampia riflessione sull'etica della vita buona, individua nella letteratura (ma più di recente anche nella musica) lo strumento e il luogo dell'apprendimento dei sentimenti di immedesimazione, compassione, dignità che conducono alla "simpatia", come capacità di pensare il bene degli Altri, componente integrante di una concezione politica basata sulla qualità della vita ${ }^{11}$.

Seppure distanti e a tratti in opposizione tra loro, i vari versanti del movimento americano convergono in definitiva sul fine della "Law and Literature Enterprise"12 impresso nella matrice originaria di questi studi, ovvero sull'educazione alla democrazia e sul progetto di un'etica pubblica, al quale evidentemente devono essere informati innanzi tutto coloro che si preparano ad amministrare la legge. Un obiettivo che si sta manifestando sempre più come il principale, soprattutto in questi ultimi anni, dopo che, nonostante la forte crescita negli ambienti della ricerca e un certo impatto anche sul discorso politico, negli Stati Uniti Law and Humanities non ha

${ }^{10}$ Ci riferiamo ancora a Posner (2009, p. 6), del quale il posizionamento critico rispetto alla più larga parte del movimento è segnato in particolare dalla presenza nel volume di un Manifesto di Law and Literature, con lo scopo di tracciare una "breve lista di precetti teoretici, metodologici e pedagogici per guidare i prossimi sviluppi di diritto e letteratura come un campo interdisciplinare di insegnamento e studio" (2009, p. 545).

${ }^{11}$ Il nucleo centrale delle opere etiche di Nussbaum sembra essere verosimilmente la ricerca di un ideale di società liberale e delle possibilità concrete di applicazione dei principi di eguale dignità e libertà di tutti i cittadini. Ciò emerge dall'analisi della natura umana che l'autrice pone come presupposto epistemologico per ogni trattazione etica e politica, analisi che prende le mosse dal pensiero aristotelico per arrivare alle teorie psicologiche contemporanee di Donald Winnicott e politiche di John Rawls e Amartya Sen.

${ }^{12}$ L'espressione riprende il titolo del famoso saggio di apertura del primo volume dello Yale Journal of Law \& Humanities (Weisberg, 1989). 
inciso significativamente sul cambiamento della logica formale che permea la legge ${ }^{13}$.

Alla domanda "How can literature have anything to say to lawyers?" si continua a rispondere in ultima istanza che dalla letteratura riprende vita la possibilità di impadronirsi dell'autentico significato di parole come responsabilità, alterità, comunità politica. Poiché il cuore della giustizia è etico e relazionale: attiene all'attitudine e alla capacità con cui i testi autoritativi sono letti e interpretati; nel tipo di attenzione data alle rivendicazioni opposte e alle esperienza di parti opposte; nella qualità dell'apertura (o della chiusura) verso nuove formulazioni, nuove voci; nel convincimento che l'opinione giudiziale o giuridica è un testo etico e politico così come intellettuale, e che soprattutto colui che lo compone ne è il principale responsabile.

\section{L'ESPERIENZA EUROPEA}

Fino a una ventina di anni fa, la storia meno nota di Diritto e letteratura è invece quella degli studi europei, radicati in una tradizione inaspettatamente estesa e articolata, risalente quasi quanto quella americana $^{14}$.

Grazie al notevole lavoro di ricognizione di Arianna Sansone (2001), scopriamo perciò che non tanto più tardi di John Wigmore (1908) o di Benjamin N. Cardozo (1924) ${ }^{15}$, in Italia Ferruccio Pergolesi pubblica, per l'appunto nel 1927, un primo articolo intitolato Il diritto nella letteratura, e Antonio D’Amato nel 1936 dà alle stampe un volume su La letteratura e la

\footnotetext{
${ }^{13}$ Lo sottolinea Paul Heald (2009) in un intervento in cui, dopo aver assunto l'impossibilità per il sistema logico-formale della legge di cambiare nelle proprie strutture portanti, precisa che il valore di Law and Literature rimane nelle proprie potenzialità educative, per maturare le qualità indispensabili all'etica, punto cardine del dibattito che sta investendo negli ultimi dieci anni la riflessione americana.

${ }^{14}$ Convenzionalmente si suole individuare l'inizio di Diritto e letteratura dal momento in cui vengono introdotti studi esplicitamente dedicati al binomio "Diritto e letteratura" ("Droit et Littérature", "Recht und Literatur", "Derecho y Literatura").

${ }^{15}$ Il riferimento è alle due prime opere a cui si fa risalire la nascita di Law and Literature negli Stati Uniti, rispettivamente di J. Wigmore, A List of Legal Novels (1908), in cui vengono selezionati e sistematizzati molteplici romanzi della narrativa moderna, di area per lo più anglosassone, interessati da tematiche giuridiche; e di B.N. Cardozo, Law and Literature (1924-1925), in cui si esaminano le qualità letterarie del diritto, prefigurando la possibilità di leggere e interpretare le sentenze come esempi di letteratura.
} 
vita del diritto ${ }^{16}$; mentre negli stessi anni in Germania Hans Fehr (1923, 1931, 1936) si occupa in modo esteso del rapporto tra diritto e arte, lavorando non solo allo studio dei soggetti giuridici nella letteratura ma anche nelle arti figurative, e Radbruch (1938) ricotruisce, mediante un'analisi comparativa tra opere rappresentative della cultura di diversi paesi europei, il sentimento giuridico delle nazioni. Vi apprendiamo inoltre che, a partire dagli anni ' 50 , la sensibilità verso questo approccio di ricerca comincia a trovare precise testimonianze anche in Francia e in Spagna (Sansone, 2001). Per aggiornare il quadro basterà aggiungere che dal 2000 in avanti, l'interesse si accresce ulteriormente in tutta Europa, estendendosi in particolare nei paesi del Nord ${ }^{17}$.

Mancano nella ricostruzione della Sansone (2001) studi di Diritto e letteratura svolti dal lato dei letterati. Evidentemente potrebbero non risultare di immediato interesse o visibili a lei che è una ricercatrice di area giuridica. Nei lavori riscontrati, tuttavia, sono tendenzialmente assenti anche forme di collaborazione tra giuristi e studiosi di letteratura, dato che parrebbe testimoniare il fatto che, diversamente da quanto succede negli Stati Uniti (soprattutto dopo la "rinascita" degli anni '70, quando il campo diventa autenticamente interdisciplinare), in Europa, Diritto e letteratura sia preso in carico per lo più dai giuristi, che elaborano una metodologia del tutto autonoma dal confronto che potrebbe provenire loro dal côté letterario. Tutto ciò almeno fino agli anni '9o.

Non che a questo corrisponda un radicamento all'interno della cultura giuridica. Ben lontano dall'essere istituzionalizzata come materia di insegnamento universitario, in Europa i lavori che emergono sotto

\footnotetext{
${ }^{16}$ Mentre l'articolo di F. Pergolesi (1927) è soltanto il primo di vari saggi che culminano nel suo più esteso lavoro - Diritto e giustizia nella letteratura moderna narrativa e teatrale (1956) -, l'unico lavoro di D'Amato cui si ha notizia è La letteratura e la vita del diritto (1936).

${ }^{17}$ Si segnala in tal senso la presenza del Nordic Network for Law and Literature tra il 2005 e il 2009 presso l'Università di Bergen (littrett.uib.no/index.php?ID=Nettverk\&lang=Eng) e dello European Network for Law and Literature introdotto in contemporanea e diretto da Jeanne Gaakeer e Greta Olson, presso la Law School di Rotterdam (eurnll.org), che si pone come obiettivo il raccordo delle varie esperienze che si vanno maturando nel campo di Diritto e letteratura in Europa. Un'ulteriore testimonianza dello sviluppo europeo degli studi di Law and Humanities la si può riscontrare nella membership oltre che dai lavori prodotti dalla Italian Society for Law and Literature (lawandliterature.org) a cui partecipano studiosi provenienti da moti paesi europei, oltreché dalla scienza internazionale più vasta, v. infra $\S 3 \cdot 3$.
} 
l'epigrafe di Diritto e letteratura nel corso del '900 non trovano grande riconoscimento nemmeno al livello della ricerca e restano per lo più esperienze individuali e isolate, senza valicare, tranne che in rare eccezioni, i confini nazionali.

Eppure, sebbene non esistano forti contatti, nulla che possa far pensare a un "approccio giusletterario europeo", questi studi manifestano tendenze comuni. Innanzi tutto, l'asse della ricerca non si sposta dai temi classici della storia del pensiero giuridico. Pur nella specificità dell'accostamento alla letteratura o ad altre arti, si esplorano i consueti problemi del fondamento del diritto, della legge o della giustizia; dell'origine degli ordinamenti giuridici; delle ragioni che presiedono al contenuto di nozioni e istituti normativi; del concretizzarsi dell'esperienza giuridica nel proprio essere storico; del rapporto tra diritto e morale, religione, potere.... Vale a dire, ed è un altro segno che li accomuna, che Diritto e letteratura è percepito negli studi europei non come una materia a sé, ma come una via ulteriore da percorrere per approfondire la riflessione giuridica, soprattutto quando si rivolge al rapporto complesso tra il diritto e l'uomo con le sue realtà. Mentre, dunque, negli Stati Uniti Law and Literature ha come principale obiettivo di incidere nel progetto democratico e "rinasce" quanto meno a partire dagli anni '70 ambiziosamente "interdisciplinare", i Diritti e letteratura europei, essenzialmente di "parte giuridica", si servono di questa prospettiva essenzialmente per la ricerca e in modo del tutto autonomo, mentre l'obiettivo della formazione giuridica sensibilizzata all'impegno politico così cruciale nell'esperienza americana - non è assolutamente in primo piano. Tra gli studiosi europei persiste più probabilmente l'idea che l'educazione al diritto e civica non è tanto un fine, quanto un'ovvia conseguenza della formazione complessiva della persona, che "si coltiva" rivolgendosi in modo ampio della cultura umanistica e scientifica, alla quale a maggior ragione si dedica il giurista per propria sensibilità, complice anche la sua vicinanza alla legge che lo obbliga ad attraversare vari mondi.

Varrebbe la pena chiedersi se queste tendenze comuni non affiorino da una memoria che abbiamo dimenticato e in cui forse si conserva un'identità che dovremmo recuperare. La scienza giuridica europea presenta una stratificazione culturale così antica e variegata che potrebbe 
non soltanto spiegare le ragioni delle particolarità nell'accostare i problemi degli studiosi europei, ma anche fornire chiavi per contributi originali.

Si consideri per esempio l'antica presunzione di sapienza che legittima i responsa dei giuristi medievali. Come ricorda Diego Quaglioni, presentendo quella che sarà la "disputa delle arti” nella temperie del primo umanesimo, Bartolo da Sassoferrato mantiene alla scienza giuridica il primato di "vera filosofia" in virtù della propria sapienza nel misurare la realtà inglobando altri saperi: "[I]l giurista è «lapis in caput anguli», chiave di volta, pietra angolare di un'architettura che è dottrinale e sociale, scientifica e politico-religiosa ad un tempo". Si tratta della "grande «opzione per la lacuna», che caratterizza il pensiero giuridico premoderno", precisa Quaglioni (2005, p. 42-44), e ancora: "nel difetto o nel silenzio delle norme giuridiche", per giungere alla "verità del diritto" il giurista medievale "allega le auctoritas morali", ovvero "integra" la propria interpretazione del caso concreto facendo ricorso a saperi ausiliari extralegali, tra cui le autorità letterarie occupano una posizione di grande rilevanza. Certamente, il processo della differenziazione delle arti e della loro professionalizzazione non si arresta, ma questa sensibilità che si rivolge alla ricerca della sapienza potrebbe non aver abbandonato il giurista europeo, quasi fosse una componente genetica della propria cultura. D'altra parte, le testimonianze di giuristi che continuano anche nel corso della modernità a coniugare al diritto conoscenze e sensibilità maturate tramite altri saperi e arti sono innumerevoli ${ }^{18}$. Inoltre, quanto non gioverebbe, ragionare ancora su un' “opzione per la lacuna”? L'idea della mancanza è una chiave potente per la critica di uno dei miti del sistema giuridico moderno, della completezza appunto, ma potrebbe essere anche una via per accrescere la sensibilità del giurista circa la propria limitatezza, specialmente nella sua principale funzione che consiste nella ricerca della misura. Se poi a "colmare la lacuna" dell'interpretazione giuridica della realtà è la letteratura, si comprende bene che la lezione per chi ambisce a occuparsi di Diritto e letteratura diventa più ampia e profonda.

\footnotetext{
${ }^{18} \mathrm{Ne}$ citiamo due a titolo di esempio. La ricerca di Jean Bodin della fine del '50o, che segue il filo della teoria musicale per elaborare un modello di "justice harmonique", e il lavoro dei fratelli Grimm, che nell'88oo ricostruiscono le consuetudini e i valori del popolo tedesco, raccogliendo le fiabe della tradizione conservate nella cultura popolare.
} 
In breve, la diversità tra le due esperienze, americana ed europea, andrebbe colta non soltanto nella differenza tra i sistemi di common e civil law - che pure non va sottovalutata (Vespaziani, 2012) -, ai quali corrisponderebbero mentalità, culture e problematiche giuridiche distanti, ma nelle tradizioni che caratterizzano due differenti storie della cultura giuridica occidentale.

Purtroppo, la specificità europea non soltanto non è stata messa ancora adeguatamente in rilievo ${ }^{19}$, ma al contrario sin dalla presa di contatto con gli studi statunitensi negli anni '9o si registra la tendenza opposta verso l'“americanizzazione” della ricerca, che indebolisce le potenzialità euristiche degli studi europei.

Di tutto ciò è causa certamente il tradizionale riconoscimento istituzionale che Law and Literature trova negli Stati Uniti, e che rende in certa misura esemplare l'esperienza americana, rispetto all'accreditamento ancora modesto di questi studi nelle nostre università. Ma questa è a nostro giudizio soltanto la ragione più superficiale.

La tendenza a mutuare temi e metodologia di ricerca dall'esperienza statunitense è parte di un processo culturale più complesso. Nell'era della globalizzazione i diversi mondi culturali e i loro problemi si accostano progressivamente fino a sovrapporsi, e così anche, di conseguenza, i modelli del diritto e delle scienze.

Complessità crescente, burocratizzazione e tecnicizzazione del diritto, deresponsabilizzazione degli operatori giuridici, incapacità di visione, di prospettiva e di reale innovazione del sistema... sono tutti problemi che accomunano l'esperienza americana ed europea e che, quindi, guidano la riscoperta dei Diritto e letteratura europei nel segno di quelli statunitensi, focalizzandosi sulla necessità di contrastare le esasperazioni del formalismo giuridico, promuovendo un ritorno del diritto alla realtà umana e sociale, e il progressivo depauperamento della capacità del giurista di coltivarsi, nel segno di un necessario progetto civico e politico che non ha più bandiere (Nussbaum, 2010).

\footnotetext{
19 Come rileva G. Olson (2010), nel senso che, pur limitando la propria analisi alla comparazione tra le esperienze statunitense e inglese e tedesca, l'A. tenta di aprire nuove prospettive di ricerca.
} 
Il fenomeno è facilmente osservabile a proposito del più recente interesse per la testualità e la culturalità del diritto, che emerge negli studi europei di rimando alle indicazioni della Law and Literature Enterprise orientate dalle acquisizioni teoriche delle scienze sociali e della comunicazione. Sono queste, infatti, le discipline più tipiche della globalizzazione culturale in cui sfumano le differenze tra cultura europea e statunitense.

Il punto è che questo tendere all'indifferenzazione e a comprimere la riflessione limitandola al tempo presente non porta alla costruzione di alternative. Quindi, pur non eludendo il confronto con l'esperienza americana, che continua a essere fondamentale e anzi dovrebbe essere approfondito, ciò che auspichiamo è di individuare la "differenza" dell'approccio europeo, perché si possa procedere da una fruttuosa "giusta distanza”. Che poi sarebbe il modo più autentico per contrastare all'origine quell'uniformità che il mondo contemporaneo impone e tanto viene osteggiata (spesso soltanto retoricamente) anche da molti cultori di Diritto e letteratura.

Una nuova fase, europea, dovrebbe pertanto essere orientata al recupero dei punti di forza della nostra cultura che potrebbero restituirci una profondità di sguardo utile all'avanzamento della ricerca nel suo complesso. Allo stesso tempo bisognerebbe rispondere alla necessità di avviare una riflessione importante di carattere metodologico (e in questo la lezione che proviene dal dibattito statunitense è cruciale) per intessere un dialogo ormai ineludibile tra studiosi del diritto, teorici della letteratura e scienziati del testo ${ }^{20}$.

\footnotetext{
${ }^{20}$ Esemplare in tal senso, nella scena europea, è il pionieristico lavoro di François Ost che promuove nei primi anni del 2000 la collaborazione tra il SIEJ (Séminaire interdisciplinaire d'études juridiques) e il SIRL (Séminaire interdisciplinaire de recherches littéraires), istituiti presso le Facultés Universitaires di Saint-Louis a Bruxelles, con l'obiettivo di operare un concreto avanzamento teorico e metodologico mettendo a confronto filosofi del diritto, giuristi e studiosi della letteratura e creando condizioni di apertura e articolazione nei reciproci modi di ragionamento. L'esperienza confluisce nel poderoso collettaneo F. Ost, L. Van Eynde, Ph. Gérard et M. van de Kerchove (2001). In seguito, Ost avanza le proprie tesi in Raconter la loi. Aux sources de l'imaginaire juridique (2004), ora parzialmente tradotto in Mosè, Eschilo, Sofocle (2007). Più recentemente, Ost è ritornato sul metodo in La justice, ses alternatives et ses symboles. Venger, pardonner ou juger? Variations littéraires (2014).
} 


\section{L'ESPERIENZA ITALIANA DI DIRITTO E LETTERATURA}

L'esperienza italiana di Diritto e letteratura nel corso del '900 riflette tendenzialmente quella europea, anche nella tensione più recente verso quella che abbiamo definito come "americanizzazione" della ricerca. Essendo, tuttavia, per noi un osservatorio privilegiato, vale la pena soffermarvisi per cogliere alcune particolarità. Diversamente che per il quadro europeo, qui riusciamo, infatti, almeno in parte a individuare anche il versante letterario degli studi di Diritto e letteratura, e ciò è importante per riflettere con maggiore cognizione sulle strade che si prestano a essere percorse.

Ce ne occuperemo a partire dagli anni '9o, da quando il successo di questi studi nel nostro paese comincia a crescere in modo esponenziale, tanto da far pensare anche a una "rinascita" italiana di Diritto e letteratura.

Per certo, la maggiore visibilità di Diritto e letteratura si registra ancora sul côté giuridico piuttosto che su quello delle lettere e delle scienze del testo. A partire dal 2000, infatti, sono più numerosi gli scienziati del diritto che, contestualmente al recupero di illustri antecedenti, guardano alle potenzialità euristiche e di carattere critico dell'approccio, e ampliano l'indirizzo sul versante delle Humanities. E sono ancora i giuristi ad ambire a uno spazio istituzionale per la materia, mettendo talvolta in campo anche il fine più ambizioso di contribuire alla crescita della democrazia curando la formazione all'etica nelle aule accademiche. Come dicevamo, è possibile osservare, tuttavia, che l'interesse per l'accostamento è presente anche tra gli studiosi di critica letteraria e del linguaggio, anzi, sembrerebbe addirittura in lieve anticipo sulla "rinascita" di Diritto e letteratura tra i giuristi. Il punto è che le diverse esperienze restano a lungo confinate nei propri ambiti disciplinari, distinte quasi del tutto per obiettivi conoscitivi e metodologie, tanto da far rinvenire in Italia due storie di Diritto e letteratura che ancora stentano a confluire l'una nell'altra. A queste se ne aggiunge una terza, la più attuale, in cui si registra, fortunatamente, l'emergere dell'incontro tra giuristi, letterati e scienziati del testo, che pur essendo ancora alle prime battute conta già esperienze importanti e di cui tenteremo di dar conto in questo contributo, anche se nei limiti della parzialità ineludibile di questo genere di operazioni. 


\subsection{La rinascita nell'ambito giuridico}

Inteso come un vero e proprio indirizzo di studi, Diritto e letteratura ricompare in Italia negli ultimi venti anni prima di tutto tra i filosofi del diritto. Che Arianna Sansone (2001) si addottori in questa materia sotto la guida di Mario A. Cattaneo non è un dettaglio ${ }^{21}$, così come non lo è il fatto che la prima cornice accademica a cui Diritto e letteratura viene ascritto sia quella della Filosofia del diritto, dove, nel 2002, la prospettiva trova un proprio spazio tra le teorie giusfilosofiche contemporanee (Faralli, 2002).

In seguito, l'interesse si manifesta tra gli studiosi di Storia del diritto medievale e contemporaneo. In particolare a Trento, G. Marchetto e M. Cau (2004) curano un collettaneo su Diritto e letteratura, e dal 2005-2006, su impulso dei giustoricisti tridentini, viene istituito il primo insegnamento recante l'epigrafe "Diritto e letteratura” dell'Università italiana. Nello stesso settore l'anno successivo, nel 2007-2008, a Roma Tor Vergata viene istituito anche il corso di Law and Humanities rivolto allo sviluppo dell'approccio letterario ma anche musicale e in generale artistico al diritto $^{22}$.

Contemporaneamente, Diritto e letteratura emerge anche tra gli studiosi di diritto privato comparato. Nel 2006 Barbara Pozzo organizza un convegno presso l'Università dell'Insubria, Teaching Law through the Looking Glass of Literature, che ospita insieme ai relatori italiani alcuni tra i maggiori esponenti di Diritto e letteratura sulla scena internazionale, europea (F. Ost, J. Gaakeer) e statunitense (J. B. White, R. Weisberg) ${ }^{23}$.

A partire dal 2008-2009 nascono altri insegnamenti di diritto e letteratura. Per primo nella laurea magistrale in Giurisprudenza dell'Università del Sannio con il settore scientifico disciplinare di diritto privato comparato; successivamente a Urbino nel 2009-2010 con il settore

\footnotetext{
${ }^{21}$ Mario A. Cattaneo, filosofo del diritto, si dedica già agli studi di Diritto e letteratura sin dagli anni '8o. Ricordiamo in particolare i lavori Carlo Goldoni e Alessandro Manzoni. Illuminismo e Diritto Penale (1987) e Suggestioni penalistiche in testi letterari (1992).

22 Il corso rappresenta una delle esperienze più vivaci nel panorama italiano per la ricchezza delle proposte tematiche e lo stile didattico innovativo (lawhumanitiesroma3.blogspot.it)

${ }^{23}$ I contenuti dell'esperienza sono riproposti attraverso la pubblicazione omonima degli atti (Pozzo, 2010).
} 
di filosofia del diritto nella magistrale in Sociologia della multiculturalità ${ }^{24}$; a Torino, nel 2010-2011, nella laurea magistrale in Giurisprudenza, inquadrato ancora come materia di diritto privato comparato; a Catanzaro - Università Magna Grecia - nel 2012-2013, di nuovo con il settore di filosofia del diritto nella laurea magistrale in Giurisprudenza; e da ultimo, con attivazione nel prossimo a.a. 2015-2016, presso l'Università del Molise nell'ambito delle attività formative della laurea magistrale in Scienze politiche e delle istituzioni europee, con il settore scientifico disciplinare di diritto pubblico comparato; e presso l'Università Federico II di Napoli, ancora per il corso di laurea magistrale di Giurisprudenza, con il settore scientifico disciplinare di diritto costituzionale.

Nel frattempo diventano sempre più numerose le pubblicazioni e le occasioni di incontro (piccoli convegni, seminari di studio, addirittura premi letterari per giuristi scrittori) e di formazione spesso rivolte anche ad avvocati, che coinvolgono studiosi dei diversi settori del diritto (sociologi; antropologi; antichisti; penalisti; civilisti; processualisti; lavoristi; costituzionalisti, e altri ancora).

I temi affrontati sono quelli classici della storia del pensiero giuridico europeo che si ripropongono a seconda della sensibilità o della formazione scientifica del singolo ricercatore. Al livello del metodo, in massima parte, l'accostamento alla letteratura è il più canonico, vale a dire quello ascrivibile al Diritto nella letteratura, dove il diritto, osservato attraverso la rappresentazione letteraria, viene restituito alla complessità propria e del contesto, e per questa via messo in questione. Più di sovente sono i grandi classici della letteratura mondiale le opere elette dai giuristi, ma non mancano studiosi che si occupano anche di letteratura contemporanea.

Sebbene meno numerose, sono presenti testimonianze significative anche sul versante della ricerca del Diritto come letteratura. Qui troviamo un certo sviluppo sul piano sia dell'analisi del linguaggio giuridico supportata dagli strumenti della critica letteraria, proposta da studiosi che intendono il diritto essenzialmente come un costrutto linguistico, interessati ai problemi dell'ermeneutica o coinvolti nell'analisi

\footnotetext{
${ }^{24}$ È necessario precisare che l'esperienza si conclude in un biennio nella facoltà urbinate per necessità strutturali dell'offerta formativa, nonostante gli esiti positivi (Bertocchi et al., 2011).
} 
comparatistica e nel gravoso tema della traduzione (Tuzet, 2005; Vespaziani, 2009, 2011); sia sul piano dell'osservazione del diritto come costrutto narrativo, condotta principalmente da ricercatori che hanno una formazione di carattere sociologico-giuridico (Di Donato, 2008; Mittica, 2006).

Quanto alla prospettiva Law and Humanities, non esiste ancora una riflessione approfondita sul campo di ricerca e le proprie potenzialità. D’altronde sono pochi i lavori emersi negli ambiti di Diritto e musica e Diritto e arti figurative, e per quel che riguarda Diritto e cinema, il maggiore e diffuso interesse si concretizza spesso soltanto nell'impiego dell'opera cinematografica nella didattica per introdurre studenti e operatori a temi complessi o a contesti culturali non familiari, senza che vi sia un vero approfondimento delle problematiche di carattere teorico e metodologico che si sprigionano dalla messa a confronto dei due ambiti ${ }^{25}$.

In ogni caso, Diritto e letteratura e Law and Humanities sembrano avere toccato sensibilmente le corde di molti studiosi del diritto italiani. Lo dicono la varietà dei temi e degli approcci, la numerosità degli interventi, l'impegno profuso per fortificare e istituzionalizzare questi indirizzi di ricerca. Il limite è che al grande entusiasmo non si è sempre accompagnata anche una debita accuratezza metodologica. Non soltanto non si è prestata attenzione al necessario confronto con gli studiosi dell'altro versante, ma nel proliferare degli studi non si è riflettuto ancora sufficientemente sui termini anche più generali di Diritto e letteratura. Ne emerge un certo grado di confusione sin nella definizione dell'approccio: ci riferiamo a una disciplina, a una metodologia o a un movimento? Non si è affrontato inoltre il problema della distinzione di campo tra Diritto e letteratura e Law and Humanities o dell'individuazione di direttrici di ricerca, obiettivi conoscitivi o altre finalità, con l'esito nella maggioranza dei casi di interpretare ognuno per sé un proprio "diritto e letteratura", oppure di mutuare distinzioni teoriche e metodologiche unicamente dall'elaborazione della Law and Literature Enterprise, con l'ulteriore esito di depotenziare la possibilità di elaborare un approccio in parte originale.

\footnotetext{
${ }^{25}$ Per una prima esplorazione su Law and Humanities si rimanda alle raccolte degli atti dei convegni ISLL disponibili nei dossier pubblicati nei volumi annuali degli ISLL Papers (The Online Collection of the Italian Society for Law and Literature, lawandliterature.org).
} 


\title{
3.2 L'esperienza di Diritto e letteratura nell'ambito della teoria letteraria e delle scienze del testo
}

Nonostante l'interesse per Diritto e letteratura sembra estendersi maggiormente sul versante degli studi giuridici, come già anticipato, le prime testimonianze della "rinascita" italiana si ritrovano tra gli studiosi di letteratura e di scienze del testo.

Il primo corso di Diritto e letteratura in Italia è tenuto nel 1998 da Remo Ceserani a Bologna su Letteratura e mondo giudiziario, sotto l'etichetta accademica di letterature comparate, settore in cui l'analisi del romanzo giudiziario sembrerebbe trovare anche altri riscontri (Adamo, 1999, 2005). Si tratta di un indirizzo di ricerca volto nello specifico all'avanzamento nello studio della struttura narrativa e della teoria del personaggio, grazie alla possibilità di osservare il processo giudiziario, raccontato nel testo letterario, come contesto compositivo in cui hanno rilievo non soltanto le storie, ma anche le azioni degli attori ${ }^{26}$. Dice Ceserani:

\begin{abstract}
Una delle lacune più gravi delle teorie narratologiche di tipo strutturalistico è stata a lungo proprio quella di non aver saputo elaborare una teoria convincente del personaggio. A quella teoria i romanzi giudiziari offrono il modello di un tipo di narrazione in cui quasi tutto sembra dipendere dal carattere del protagonista, un carattere che d'improvviso scopre, in un'azione gravida di conseguenze, la sua più profonda natura, le sue ambiguità psicologiche, i suoi sottofondi amletici (essendo Amleto - il sarcastico denigratore del teschio di un vecchio avvocato maltrattato dalla pala del becchino - lui stesso un esempio di non-azione, di ricerca tormentosa delle ragioni della sua non-azione, di continuo avvolgimento nella rete delle motivazioni pretestuose e casidiche di quella sua non-azione) (Ceserani, 2010, p. 162-163).
\end{abstract}

Sempre nello stesso ambito disciplinare, ma con un taglio più tradizionale, si sviluppa l'interesse a utilizzare il diritto come un indicatore di carattere storico-sociale ai fini della ricostruzione del contesto dell'opera e del suo autore. Il maggiore riferimento è il filone, ormai classico negli studi anche italiani di Diritto e letteratura, incentrato su Shakespeare e il diritto, dove la ricerca impone di coniugare all'analisi letteraria quella

\footnotetext{
${ }^{26}$ Il romanzo giudiziario è un genere messo in rilievo ancora di recente da R. Ceserani (2010), dove un intero capitolo è dedicato a Diritto e letteratura ("Giudici, avvocati, esperti nel determinare colpe e punizioni”, p. 141-164).
} 
giustoricistica, rivelando non soltanto nuovi aspetti della biografia e della personalità di Shakespeare piuttosto che del suo teatro, ma come l'opera shakespeariana influenzi la riflessione sul diritto dei propri contemporanei anche ai più alti livelli della gerarchia sociale. Ne è prova lo stretto rapporto tra l'autore e Giacomo I, successore di Elisabetta (Carpi, 2003; Pittion, 2003; Restivo, 2011).

Una declinazione ulteriore della ricerca si sviluppa nell'ambito della teoria letteraria, dove si investiga il rapporto tra la letteratura e il diritto. Dalle opere letterarie emerge una critica profonda della legge e l'osservazione della pervasività con cui la stessa investe le dimensioni fondamentali dell'esistenza umana individuale e sociale. È Claudio Magris, in un bel saggio su Diritto e letteratura (2006a, 2006b), a porre in rilievo l'avversione della letteratura verso la legge, come rifiuto della violenza (insita nella legge stessa) e al contempo riflesso della possibilità del male che è nell'uomo, tenuto sotto scacco tra bene e male, colpa e desiderio di perdono, giustizia e ingiustizia, consentendoci di cogliere la qualità della sensibilità che un letterato mette in atto nel riflettere su temi che sono classici del pensiero giuridico.

Studi di critica letteraria, che ben si presterebbero a essere identificati come di Diritto e letteratura, sono infine quelli che in modo del tutto autonomo procedono da tempo all'analisi del rapporto conflittuale tra letteratura e potere, osservato in racconti di denuncia se non più direttamente nei dispositivi di censura ${ }^{27}$, e spontaneamente tendono alle finalità più ambiziose di carattere sociale e politico che abbiamo visto essere nel DNA della Law and Literature Enterprise.

Sebbene lo sviluppo di Diritto e letteratura sembri più contenuto sul versante letterario e delle scienze del testo, le direttrici di ricerca appaiono più definite e, come vedremo, si prestano a essere complementari se non anche a coincidere in parte con gli studi del versante giuridico. Il limite, se vogliamo, è come per i giuristi quello di riferirsi prevalentemente alla propria comunità scientifica, subendo la settorializzazione della conoscenza che in Italia rispecchia non soltanto il processo di specializzazione dei

\footnotetext{
${ }^{27}$ Tra i possibili esempi, si rimanda alla raccolta di saggi curata da R. Francavilla (2009), e nell'ambito più ampio delle humanities a L. Gaudino (2007). Un'operazione interessante è da ultimo quella di A. Armano (2013).
} 
saperi tipico della scienza moderna, ma la struttura stessa della ricerca e della formazione universitarie.

\subsection{L'incontro tra giuristi e letterati}

Nonostante la tendenza dominante resti quella della separazione tra $\mathrm{i}$ diversi ambiti disciplinari, è importante sottolineare che in questi ultimi anni sta divenendo forte l'esigenza di creare varie occasioni di incontro e lavoro tra giuristi, letterati e scienziati del testo, a testimonianza di un cambiamento di corso che promette anche una maturazione per le problematiche di carattere metodologico.

I primi forse a incontrarsi "ufficialmente" sul terreno di Diritto e letteratura sono i comparatisti delle letterature e del diritto. Probabilmente quelli con maggiore attitudine e attrezzati per attraversare mondi diversi. L'esperienza più significativa è una bella ricerca sul concetto di equity, conclusa nel 2006, che raccoglie intorno a questo tema il lavoro di comparatisti della letteratura inglese e del diritto privato (Carpi, 2007) e dalla quale nel 2008 prende avvio l'Associazione Italiana di Diritto e Letteratura (AIDEL) ${ }^{28}$.

In contemporanea, ma con un progetto più ambizioso, viene fondata anche la Italian Society for Law and Literature (ISLL), presso il Centro interdipartimentale di ricerca in Storia, Filosofia e Informatica del Diritto (CIRSFID) dell'Università di Bologna. La ISLL nasce per promuovere gli studi di Diritto e letteratura e Law and Humanities creando un network internazionale e interdisciplinare - intercettando studiosi già affermati nell'ambito e altri interessati a un primo accostamento, raccogliendo le numerose esperienze isolate - attraverso cui realizzare un osservatorio privilegiato sugli studi in Europa e nel resto del mondo, porre le condizioni per un confronto con gli esponenti del movimento americano e contribuire all'accreditamento di questo indirizzo di studi nell'insegnamento e nella ricerca universitari. A testimonianza che l'iniziativa risponde a un'esigenza già più che diffusa, basterà dire che l'accoglienza incontrata dalla ISLL è

\footnotetext{
${ }^{28}$ Ispirata prevalentemente dalla metodologia anglosassone di Law and Literature, l'AIDEL è fondata nel 2008 da Daniela Carpi (studiosa di Lingua e letteratura inglese) e da Pierluigi Monateri (studioso di diritto privato comparato). Attualmente sta veicolando varie iniziative, per le quali si rimanda al sito dell'associazione, www.aidel.it.
} 
così vasta che nel giro di un anno supera le duecento adesioni dall'Italia e dall'Estero ${ }^{29}$. Quanto alla ricerca vera e propria, oltre a creare occasioni di riflessione a tema attraverso i convegni annuali della società e gli Special Workshop coordinati in seno ai congressi mondiali dell'IVR (Internationale Vereinigung für Rechts - und Sozialphilosophie), la ISLL sta attualmente promuovendo la formazione di gruppi di ricerca interdisciplinari su temi specifici, affinché il lavoro comune possa veicolare anche un'appropriata analisi del metodo ${ }^{30}$.

Non mancano seminari permanenti di Diritto e letteratura che nel volgersi alla formazione giuridica contribuiscono contestualmente alla ricerca. Ne segnaliamo due, i cui cicli di conferenze sono divenuti ormai degli appuntamenti fissi: Davanti alla legge. Immaginare il diritto, avviato nel 2006 dall'Università Suor Orsola Benincasa, in cui si alternano, nelle discussioni sul tema prescelto per il ciclo, giuristi, letterati (critici e scrittori), e quando, la presentazione concerne l'opera cinematografica, critici e registi; Giustizia e letteratura, avviato dal Centro Studi "Federico Stella” sulla Giustizia penale (CSGP) dell'Università Cattolica di Milano nel 2009, da cui sono sorte anche due importanti pubblicazioni che danno conto dell'esperienza sin qui svolta (Forti et al., 2012, 2014). In entrambi i casi la scommessa metodologica è di mettere a contatto i partecipanti, grazie alle testimonianze di relatori aventi diverse formazioni, con le interpretazioni di testi significativi pertinenti a temi giuridici, affinando così l'apertura al dialogo interdisciplinare, nonché la sensibilità culturale degli appartenenti al mondo delle professioni giuridiche e degli studenti ${ }^{31}$.

29 La ISLL attualmente conta 242 membri. Se ne trova la storia sul sito web (www.lawandliterature.org), che fa da collettore di informazioni e allo stesso tempo si va costruendo come un osservatorio sugli studi italiani e al livello internazionale. Oltre a essere in costante aggiornamento quanto a eventi e altre news, il sito informa su libri (library), enti di ricerca (link), e pubblica una collezione di saggi accessibili gratuitamente per consentire la più larga diffusione dei materiali di ricerca (ISLL Papers).

${ }^{30}$ Un esempio importante di avvio della nuova configurazione della ricerca all'interno della ISLL è il DIREL, il centro studi "Diritto, Religioni e Letterature" in collegamento con l'Università di Torino. Per maggiori informazioni si rimanda al sito direl.aliresearch.eu.

${ }^{31}$ Per visionare le esperienze nel dettaglio v. centridiricerca.unicatt.it/csgp_2224.html e unisob.na.it/eventi/eventi.htm?vr=1\&id=2091. 
Un ulteriore accostamento che si sta sviluppando sul terreno di Diritto e letteratura è quello tra giuristi e scienziati del testo interessati all'Italiano giuridico e, per così dire, all'Italiano dei giuristi. Ne è un vivo esempio il convegno svoltosi all'interno dell'edizione 2013 de La piazza delle lingue, dedicata a Lingue e diritti, e organizzato dall'Accademia della Crusca $^{32}$, insieme all'Istituto di Teoria e Tecniche dell'Informazione Giuridica del Consiglio Nazionale delle Ricerche (ITTIG-CNR)33.

Non si tratta qui dello studio del rapporto tra diritto e linguaggio, declinato tradizionalmente sui vari versanti della filosofia del diritto, ma dell'espressione linguistica della legge, un ambito di ricerca ancora prevalentemente frequentato dai linguisti (Dell' Anna, 2013), dei quali si percepisce l'avvertita apertura interdisciplinare. Precisa in tal senso Mortara Garavelli:

Di fronte allo stesso oggetto di studio le pertinenze dei due campi, linguistico e giuridico, si intrecciano e si sovrappongono; e la reciprocità delle competenze si impone con una forza che non ha riscontri [...]. A ciascuno il suo: è buona norma di igiene disciplinare tenere ben presenti le differenze di campo, che sono intuitivamente evidenti. L'occhiale del giurista scruta i fatti di lingua negli aspetti che sono pertinenti alle teorie generali del diritto, all'interpretazione e all'applicazione delle norme. L'occhiale del linguista cerca di isolare nei testi giuridici i tratti che li caratterizzano in quanto appartenenti a varietà di lingua distinte nel tempo, nella distribuzione geografica, nel mezzo di attuazione (scritto o parlato), nei registri; cerca di riconoscere i profili argomentativi, le strutture sintattiche, semantiche e testuali, le implicazioni pragmatiche, sociolinguistiche e via elencando. Giuristi e linguisti potranno essere ugualmente interessati a questioni tipologiche, a ricognizioni storiche, ai problemi degli usi che diremo patologici del linguaggio giuridico e alle controverse proposte dirette a semplificarlo. Le eventuali incompetenze verranno fuori nelle occasioni in cui gli uni,

\footnotetext{
32 È importante l'impegno dell'Accademia che già da qualche tempo si sta interessando ad ambedue i versanti, come testimoniano alcune iniziative, tra cui quelle confluite nei collettanei a cura di Federigo Bambi e Barbara Pozzo (2012) e a cura di Alarico Mariani Marini e Federigo Bambi (2013).

33 L'ITTIG-CNR svolge attività di ricerca, alta formazione, consulenza e trasferimento tecnico-scientifico nel campo delle tecnologie dell'informazione e della comunicazione applicate al diritto e alla pubblica amministrazione. In particolare si occupa di semplificazione del lessico e della composizione dei documenti giuridici. Con particolare riguardo per gli atti amministrativi, nel 2010 ha presentato un breve manuale di "Regole e suggerimenti per la redazione degli atti amministrativi", disponibile sul sito dell'Istituto, a cui si rimanda per ulteriori informazioni, ittig.cnr.it.
} 
addentrandosi nei territori degli altri, non trattino con le necessarie avvertenze, e coi dovuti sospetti, oggetti di ricerca e strumenti altrui che sembrino fatti apposta per avallare illusorie analogie (Mortara Garavelli, 2001, p. 4$5)$.

L’indicazione è importante perché chiarisce, tra i molti punti di contatto e di opportuno confronto, che il lavoro interdisciplinare non è la messa da parte delle competenze specialistiche, ma il dialogo molto più complesso messo in gioco tra prospettive diverse.

Concludiamo, infine, segnalando un nuovo indirizzo di ricerca emerso nel campo giuridico ma interessato a un'estetica del diritto costruita in modo autenticamente interdisciplinare. Si tratta di una prospettiva che porta in sé l'evoluzione di Diritto e letteratura in Law and Humanities, nel senso del superamento di una visione del diritto strettamente limitata al testo scritto, formulato linguisticamente34. L'estetica giuridica si volge all'elaborazione di tesi e categorie per cogliere nei testi della cultura la normatività di qualunque forma (siano testi scritti, iconici, musicali o riferibili alla dimensione spaziale e corporale, come la scultura o la danza), a cui si aggiunge l'ulteriore versante della riflessione sull'arte nel suo essere e divenire, come ricerca formale che viene posta in rapporto al diritto per osservare lo scarto tra i diversi modi di "dare forma" ai mondi della vita (Heritier, 2011). Su questa tematica si è svolto, in particolare, l'ultimo convegno nazionale della ISLL, nel 2014, dove si è sperimentata una forma di transdisciplinare di approccio alla ricerca che ha consentito di coniugare diversi saperi e sensibilità.

\section{DISTINZIONI DI CAMPO E ALCUNE PROPOSTE}

Da quanto sin qui emerso l'elemento di debolezza che sembra essere trasversale negli studi di Diritto e letteratura e di Law and Humanities, negli studi europei ma con particolare riferimento all'esperienza italiana, è

\footnotetext{
${ }^{34}$ Lo sviluppo di buona parte della Law and Literature Enterprise verso l'indirizzo Law and Humanities si basa d'altronde proprio sulla consapevolezza che è troppo riduttivo considerare le testualità del diritto e della letteratura restigendole al tipo di testo scritto a parole. Si pensi alla poesia che necessita di essere "cantata" con i metri impiegati, o alla pièce teatrale che perde buona parte del suo senso se non è messa in scena. E allo stesso modo all'arringa di un avvocato o alla pronuncia di una sentenza, alle intonazioni che necessitano, i registri che richiedono, a come cambia il senso a seconda del modo in cui il testo è espresso e recepito.
} 
la mancanza di un'adeguata attenzione di carattere metodologico alla quale si sta cominciando a mettere riparo soltanto in questi ultimi tempi. Nel clima complessivo, si avverte un certo grado di confusione sin nelle definizioni più generali di Diritto e letteratura, così come nelle distinzioni di campo e nei collegamenti che interessano i vari versanti disciplinari coinvolti, le cui prospettive sono ancora spesso separate.

$\mathrm{Ci}$ permettiamo quindi di proporre alcune distinzioni e alcune operazioni di carattere metodologico, che tengono conto della situazione italiana ed europea ma che speriamo possano risultare utili anche per il confronto nel dibattito brasiliano e internazionale.

\subsection{Distinzioni di campo. Il genere e la specie.}

La prima distinzione da mettere in atto, preliminare a tutte le altre, come ben si comprenderà, è quella tra Law and Humanities e Diritto e letteratura. Se il primo attiene al genere, il secondo ne è una specie, al pari di Diritto e cinema, Diritto e musica, Diritto e arte in generale. Il campo di Law and Humanities include dunque tutti i versanti disciplinari interessati dal binomio. Vale a dire che gli studiosi coinvolti saranno quelli del diritto di volta in volta in rapporto con coloro che si occupano di letteratura e lingua, cinema, musica o altre arti.

\subsection{Disciplina, metodologia o movimento?}

Una seconda distinzione procede dalla domanda se Diritto e letteratura, e a questo punto dovremmo dire meglio il campo di Law and Humanities, sia da considerarsi come un ambito disciplinare, metodologico o di approcci all'attivismo politico.

La risposta è che in Law and Humanities si riassume una metodologia di ricerca volta a far dialogare competenze specialistiche differenti interessate da ambiti comuni, con l'obiettivo di mettere a punto categorie e altri strumenti di analisi ad hoc per operare sull'oggetto specifico dell'indagine, nella prospettiva di creare un approccio transdisciplinare più che un dialogo interdisciplinare, che promette un maggiore successo quanto più riesce a coinvolgere la capacità di sguardo proveniente dalle diverse specializzazioni. 
Detto ciò, pur non connotandosi come disciplina, Law and Humanities può essere materia di insegnamento e trovare rilievo non soltanto sul versante degli studi giuridici, soprattutto quando, come abbiamo visto in particolare nel caso della letteratura e dell'analisi testuale, il rapporto delle humanities con la legge diviene serrato.

Come movimento politico, infine, Law and Humanities resta una scelta che, nello specifico, sembra più eletta tra i giuristi, ma senza che si possa escludere un coinvolgimento anche di studiosi sugli altri versanti, qualora si creasse una sensibilità comune intorno al recupero della cultura umanistica quale dimensione fondamentale per la realizzazione di un progetto democratico che ormai - ha ragione Nussbaum - interessa tutti.

\subsection{Quale posto per Diritto e Letteratura}

Quanto detto sin qui vale evidentemente soprattutto per Diritto e letteratura, che di fatto è l'indirizzo che ha avuto ovunque più riscontro nel campo di Law and Humanities, complice la lunga tradizione dell'analisi delle rappresentazioni letterarie della legge, e le tesi recenti sulla familiarità "testuale" dei documenti giuridici e letterari, essendo maggiormente diffusa un'idea di testo limitata alla composizione scritta e formulata linguisticamente.

L'obiettivo che ci si prefigge in questa sede è quindi di segnare qualche passo per avviare una riflessione di carattere metodologico che possa coinvolgere sia giuristi che letterati e linguisti.

A tal fine tenteremo di accostare i versanti delle discipline riscontrate sul lato giuridico di Diritto e letteratura, traendo spunto da alcune delle prospettive individuate da Arianna Sansone (2001)35, con quelli delle materie letterarie e delle scienze del testo in cui è visibile l'interesse per il diritto. Nel condurre questa operazione sarà utile, inoltre, tener presente la

\footnotetext{
${ }^{35}$ Si precisa che, mentre Sansone individua le proprie "principali prospettive e direzioni di ricerca" riferendosi al quadro internazionale degli studi di Diritto e letteratura aggiornato al 2001 (le prospettive in questione sono: storico-antropologica, sociologico-giuridica, filosofico-politica, della filosofia del diritto che si occupa di teoria della giustizia e della filosofia del diritto che si occupa di teoria generale del diritto - p. 111 e ss.), qui si fa riferimento essenzialmente al caso italiano e al quadro più recente degli studi che si sono notevolemente accresciuti e diversificati nell'ultimo decennio. Per cui le direzioni di ricerca indicate da Sansone sono riprese, ma riorganizzate e completate sulla base della ricognizione esposta.
} 
distinzione rilevata tra studi giuridici dal lato del diritto e studi di letteratura e scienze del testo dall'altro lato, e tra i due approcci del Diritto nella e come letteratura, ancora largamente impiegati.

Sansone indica come prime tra le materie più sensibili all'impiego dell'approccio giusletterario la storia del diritto e l'antropologia giuridica. Le competenze chiamate in campo sull'altro côté sono quelle della critica letteraria. L'approccio metodologico è del Diritto nella letteratura. A questa direttrice può essere assimilata, tuttavia, anche la sociologia del diritto quando assume tra le proprie fonti di ricerca le opere letterarie. Per storici, sociologi e antropologi giuridici, la letteratura viene in rilievo come testimonianza del pensiero e del sentire dell'uomo in relazione all'esigenza della regolamentazione normativa della convivenza sociale e politica, del passato o del presente, vicina alla propria cultura o lontana da essa, al fine di cogliere un'esperienza giuridica nella complessità del proprio contesto culturale. Lo studioso del diritto osserva attraverso l'opera letteraria (classica o contemporanea, occidentale o non) il senso giuridico comune, le regole scritte e quelle non scritte, il sentimento della legge, le visioni del sistema giuridico e dei suoi operatori, servendosene nella costruzione di ipotesi sul rapporto tra il diritto e l'uomo, la comunità, la società. Complementare a questo interesse è quello dello studioso di storia della letteratura o di letterature comparate che intende servirsi del diritto come indicatore per comprendere il contesto di un'opera e del suo autore, essendo il diritto (inteso sia come legge che come diritto informale) un fattore rappresentativo e di grande influenza delle culture e delle società di ogni dato periodo storico. Evidentemente, così come è difficile per i giuristi padroneggiare l'interpretazione di un testo letterario in modo scientifico non possedendo appunto gli strumenti dell'analisi letteraria - allo stesso modo è consigliabile ai letterati di rivolgersi agli studiosi del diritto per farsi guidare nella comprensione dei fenomeni giuridici, essendo necessarie anche su questo fronte competenze molto sofisticate. La migliore soluzione sta nel raccogliere le diverse abilità intorno all'individuazione dell'opera, dell'autore e del contesto, come terreno comune di indagine in modo che, pur nella differenza degli obiettivi conoscitivi, sui due versanti si possa operare la selezione delle informazioni più utili a ciascuno per il proprio 
avanzamento e contestualmente essere coadiuvati nell'interpretazione dei dati in esame.

Qui si tratta, in altre parole, di mettere in opera un lavoro interdisciplinare. Molto semplice a dirsi nella sua formulazione verbale, ma estremamente complesso da gestire nella pratica, quando appunto entrano in gioco gli specialismi e bisogna incontrarsi in un terreno di "traduzione" e "mediazione".

Le stesse indicazioni possono essere prestate in via generale a tutte le direttrici di ricerca che andiamo a riassumere qui di seguito, sebbene quando l'approccio metodologico è del Diritto come letteratura, il lavoro interdisciplinare si presta a sfociare in una ancor più preziosa transdisciplinarietà.

È questo delle ulteriori prospettive di ricerca che si ascrivono alla sociologia del diritto: 1) quella che osserva il diritto essenzialmente come un fenomeno comunicativo, e 2) quella che ne osserva il contesto in termini di costruzione narrativa. Le competenze chiamate in campo nell'approccio giusletterario sull'altro côté sono, in questo caso, delle scienze del testo e dell'analisi del linguaggio giuridico. L'approccio metodologico è del Diritto come letteratura.

Osservato come fenomeno comunicativo, il diritto è un complesso di messaggi giuridici. In questa prospettiva, per qualunque problema si voglia affrontare (funzioni, efficacia, impatto, ecc.) è necessario comprendere la norma nella propria qualità di costrutto simbolico, condizionato dai meccanismi di codificazione e composizione del testo, dai vari canali nel processo di comunicazione, così come dai problemi connessi alla ricezione $\mathrm{e}$ alla decodificazione del messaggio (Ferrari, 2013). La complementarietà degli studi che si occupano di lessico e composizione dei testi giuridici dal lato letterario del binomio è evidente. Per taluni aspetti, inoltre, le finalità conoscitive degli scenziati del testo sembrano anche coincidere con quelle dei sociologi. Si pensi, p.e., alla necessità di osservare il passaggio dal piano tecnico-formale del linguaggio legislativo al piano della traduzione di senso nel linguaggio comune, per comprendere quale ricezione del messaggio abbiano avuto i destinatari. Qui, infatti, se ai sociologi sarebbe utile un'osservazione tecnica dei costrutti linguistici (p.e. per comprendere lo scarto tra il linguaggio impiegato nella formulazione giuridica e quello del 
comune cittadino), al linguista potrebbe servire comprendere altri elementi che intervengono nel processo comunicativo (p.e. le caratteristiche dei canali di trasmissione del messaggio quando sono in particolare operatori giuridici) o i meccanismi di funzionamento del sistema giuridico o ancora le prassi che influenzano la composizione dei testi giuridici.

Dalla prospettiva che osserva il contesto sociale in termini di costruzione narrativa, invece, il diritto è esso stesso un costrutto narrativo. La prospettiva, poco frequentata in passato dalla sociologia del diritto italiana, si è sviluppata di recente in stretto rapporto con la sociologia narrativa e la psicologia culturale (Jedlowski, 2000; Smorti, 2007). La tesi di fondo ricalca quelle del Law as Narrative americano, ovvero l'idea che, essendo le narrazioni i processi costitutivi delle relazioni, dunque della stessa società, le comunità prendono vita e si conservano tramite la condivisione di racconti (Ost, 2004). Più nello specifico, gli studi italiani si concentrano sulle dinamiche narrative che interessano al pari di altri racconti il diritto e la legge nella costruzione della realtà sociale. E non si tratta di comprendere soltanto l'immagine che la legge o il sistema giuridico dà di sé, ma di penetrare nella costruzione dell'immaginario giuridico comune: dove i racconti giuridici vengono scambiati, nascono aspettative, si intraprendono e condividono lotte, si attendono funzioni che il diritto non sempre può assolvere o che restano latenti. A questo profilo si accosta il lavoro degli studiosi che si occupano delle strutture narrative dei testi letterari in cui si impiegano dispositivi giuridici reinterpretandoli in chiave narrativa. Dalla ricognizione effettuata, si presta come esempio fecondo l'analisi del romanzo giudiziario. Qui il terreno comune per giuristi e studiosi del testo è per eccellenza costituito dal processo giudiziario nei termini in cui viene impiegato per veicolare una storia che racconta sempre della rottura di ordine consolidato, del disagio che ne consegue e del ripristino di un equilibrio. Il processo è il dispositivo più interessante per veicolare tutti gli elementi che prendono parte e incidono nel corso del ripristino dell'ordine. Se l'analisi narratologica è volta a comprendere come attraverso il processo giudiziario un autore costruisce e risolve la narrazione (si pensi alla funzione narrativa svolta da questo dispositivo in moltissimi classici della letteratura), lo studioso del diritto potrebbe acquisire dalla stessa analisi alcune chiavi per svelare le funzioni più latenti dei processi. 
Sempre in linea con l'approccio metodologico del Diritto come letteratura, mettendo in gioco tra le direttrici di ricerca ascrivibili a Diritto e letteratura la prospettiva giusfilosofica che si occupa di analisi del linguaggio giuridico, Sansone ci induce a chiamare i linguisti sul côté della teoria generale del diritto. Di fatto, nelle tendenze attuali degli studi, non abbiamo rilevato ancora una collaborazione di carattere interdisciplinare. Tuttavia, se, come ricorda Dell'Anna (2013, p. 17), “[I]l punto di contatto tra i due interessi scientifici e professionali è stato ed è tutt'ora il livello semantico", e la separazione tra giuristi e linguisti è altrettanto netta, elaborando i primi "una prospettiva teorico-interpretativa", e i secondi una "testuale e comunicativa", c'è da chiedersi quali opportunità potrebbe invece offrire una condivisione di campo. Si pensi p.e. alla ricerca svolta dai linguisti con l'obiettivo della "semplificazione" del linguaggio giuridico. "Semplificare", non è soltanto un togliere, un alleggerire, un riportare all'Italiano la lingua del diritto, è anche "chiarire" il dettato della norma, e ciò non può essere lasciato ai linguisti. In questo caso, l'intervento dei filosofi analitici non serve soltanto per portare a migliori risultati, ma soprattutto per diminuire il rischio della distorsione del senso. Con questo vale anche il contrario. Sono altrettanto evidenti i numerosi vantaggi che anche teorici generali del diritto possono trarre impiegando dagli strumenti messi a disposizione dai linguisti per l'analisi testuale e del linguaggio, un esempio per tutti l'apparato metodologico per lo studio delle metafore.

Tra le materie più sensibili all'impiego dell'approccio giusletterario indicate da Sansone c'è infine la filosofia del diritto. Le competenze chiamate in campo sull'altro côté sono quelle della critica letteraria, mentre l'approccio metodologico è del Diritto nella letteratura. Qui, temi e obiettivi conoscitivi sono in parte addirittura coincidenti. I problemi che coinvolgono i critici letterari sono presi in carico dai filosofi del diritto e viceversa. Dall'analisi sulla relatività, l'incertezza, l'ambiguità della giustizia umana a fronte della continua tensione verso un assoluto della giustizia che metta a riparo l'uomo dalla sua attrazione per il male e gli sconfinamenti, dall'ineludibile senso di colpa, alla riscoperta della natura corporea dimenticata del diritto (e del suo rifiuto del desiderio, della materialità), alle analisi che commentano gli universali giuridici sottesi alla convivenza sociale, nonché a quelle tese ad affermare i contenuti e i valori dell'etica 
giuridica con l'obiettivo di progettare un diritto a misura d'uomo: in questo campo le opere letterarie e artistiche permettono di abitare non soltanto la complessità della giustizia come limite e misura delle azioni umane, ma di sostare anche nello spazio del non dicibile, nel silenzio di Dike come già troviamo in Solone e non a caso in uno dei suoi frammenti poetici (Noussia, 2001). Pertanto, pur non accettando la provocazione di Posner (2009), quando afferma che la filosofia del diritto potrebbe essere sostituita dalla grande letteratura, non si può negare la fecondità di questo approccio che è capace di tenere insieme due sensibilità che ben potrebbero affinare ulteriormente le proprie categorie interpretative ed elaborarne di nuove in una chiave transidiciplinare se messe adeguatamente a contatto.

\section{IN-CONCLUSIONE}

In-conclusione vuol dire che nel chiudere questo contributo sullo stato dell'arte degli studi di Diritto e letteratura in Europa e in Italia non c'è nulla di concluso. A fronte di un quadro così variegato e in rapido mutamento, mi sia consentito dunque il gioco di parole, per riassumere più che altro la complessità di questo orientamento di ricerca con l'avvertenza ulteriore che l'accostamento a questi studi necessita forse anche di una maggiore accortezza, perché troppo spesso la familiarità e la passione per le humanities rischia di pregiudicare la giusta distanza e la cautela metodologica che sono sempre necessarie nella ricerca scientifica.

Si tratta infatti di una partita molto seria che va giocata con attenzione e capacità critica. Una partita in cui si accoglie l'invito a seguire la riflessione che procede nel dibattito americano, ma si richiede di recuperare anche una sensibilità culturale che è propria della tradizione europea e potrebbe offrire un contributo originale.

La scommessa si riversa principalmente sul piano metodologico, dove si giocherebbero probabilmente gli aspetti più significativi dell'incontro tra le due culture e che è al contempo un necessario fronte di lavoro per sviluppare una metodologia interdisciplinare, ancora tendenzialmente assente dalla nostra ricerca, da volgere in transdisciplinarietà.

E ancora è cultura. Perché queste forme di approccio metodologico non consistono soltanto in tecniche, ma divengono spazi di incontro di diverse identità che mettono in comune le proprie specificità, che chiedono 
l'umiltà di chi si pone in relazione con un "altro". Una cultura della differenza che va salvaguardata, contro una globalizzazione in corsa verso l'appiattimento dello sguardo su un unico orizzonte. In fondo siamo nel solco più originario della ricerca e della conoscenza.

Responsabilità, alterità, linguaggio, sono le parole che più insistentemente ricorrono nei lavori di Diritto e letteratura. Bisogna soltanto farsene carico seriamente.

\section{RIFERIMENTI BIBLIOGRAFICI}

ADAMO, S. Il cappello del prete: il romanzo giudiziario e il racconto della giustizia. In: CREMANTE, R. (Ed.). Emilio De Marchi un secolo dopo. Roma: Ed. di Storia e Letteratura, 2005. p. 125-156.

ADAMO, S. Mondo giudiziario e riscrittura narrativa in Italia dopo l'Unità. Problemi, n. 113, p. 70-98, gen.-apr. 1999.

ARMANO, A., Maledizioni. Processi, sequestri e censure a scrittori ed editori in Italia dal dopoguerra a oggi, anzi domani. Materiale giudiziario. Torino: Nino Aragno 2013.

BAMBI, F.; POZZO, B. (Eds.). L'italiano giuridico che cambia. Atti del Convegno di studi, Firenze, $1^{\circ}$ ottobre 2010. Pisa: Pisa University Press, 2012.

BERTOCCHI, M.; BISCI, E.; CHIUSELLI, L.; COPOOLA, V.; MAGGIO, M. C.; ORAZI, M.; PONTI, G.; RUBINO, U. D(i)ritti fra le righe. Esperienze dal corso di "Diritto e letteratura", Università di Urbino, a.a 2009-2010. ISLL Papers, v. 4, 2011.

BINDER, G.; WEISBERG, R. Literary Criticism of Law. Princeton: Princeton University Press, 2000.

BROOKS, P. Law and its Other in Literary Theory. Frame, v. 24, n. 1, p. 3252, 2011.

BRUNER, J. La fabbrica delle storie. Letteratura, Diritto, Vita. Roma-Bari, Laterza 2002.

BRUNER, J. The Narrative Construction of Reality. Critical Inquiry, v. 18, p. 1-21, 1991.

BRUNER, J.; AMSTERDAM, A. Minding the Law. Cambridge (MA): Harvard University Press, 2000.

CARDOZO, B. N. Law and Literature. Yale Review, v. 14, p. 699-718, 19241925 .

CARPI, D. (Ed.). The Concept of Equity. An Interdisciplinary Assessment. Heidelberg: Universitätsverlag Winter, 2007.

CARPI, D. Shakespeare and the Law. Ravenna: Longo 2003.

CATTANEO, M. A. Suggestioni penalistiche in testi letterari. Milano: Giuffrè, 1992. 
CATTANEO, M. A. Carlo Goldoni e Alessandro Manzoni. Illuminismo e Diritto Penale. Milano: Giuffrè, 1987.

CESERANI, R. Convergenze. Gli strumenti letterari e le altre discipline. Milano: Bruno Mondadori, 2010.

COVER, R. The Supreme Court, 1982 Term. Foreword: "Nomos and Narrative". Harvard Law Review, v. 97, v. 1, p. 4-68, nov. 1983. Tr. it. Nomos e narrazione. Una concezione ebraica del diritto. Torino: Giappichelli, 2008.

D’AMATO, A. La letteratura e la vita del diritto. Milano: Ubezzi \& Dones, 1936.

DELL'ANNA, M. V. In nome del popolo italiano. Linguaggio giuridico e lingua della sentenza italiana. Formello (RM): Bonacci, 2013.

DI DONATO, F. La costruzione giudiziaria del fatto. Il ruolo della narrazione nel "processo". Milano: Franco Angeli, 2008.

EWICK, P.; SILBEY S. S. Subversive Stories and Hegemonic Tales: Toward a Sociology of Narrative. Law \& Society Review, v. 29, n. 2, p. 197-226, 1995.

FARALLI, C. La filosofia del diritto contemporanea: $i$ temi e le sfide. Roma-Bari: Laterza, 2002.

FEHR, H. Die Dichtung im Recht. Bern: A. Francke, 1936. v. 3.

FEHR, H. Das Recht in der Dichtung. Bern: A. Francke, 1931. v. 2.

FEHR, H. Das Recht im Bilde [Kunst und Recht]. Bern: A. Francke, 1923. v. 1.

FERRARI, V. Lineamenti di sociologia del diritto. Roma-Bari: Laterza, 2013.

FORTI, G.; MAZZUCATO, C.; VISCONTI, A. (Eds.). Giustizia e Letteratura I. Milano, Vita e Pensiero, 2012.

FORTI, G.; MAZZUCATO, C.; VISCONTI, A. (Eds.). Giustizia e Letteratura II. Milano, Vita e Pensiero, 2014.

FRANCAVILLA, R. Leggere la cenere. Saggi su letteratura e censura. Roma: Artemide, 2009

GAUDINO, L. Cinema alla sbarra. Trent'anni di avventure e sventure giudiziarie del cinema italiano. Udine: Forum, 2007.

HEALD, P. The Death of Law and Literature. Research Paper Series, University of Georgia, School of Law, Paper n. 09-006, 2009.

HERITIER, P. La terza E: epistemologia, ermeneutica, estetica giuridica. In: MITTICA, M. P. (Ed.). Diritto e narrazioni. Temi di diritto, letteratura $e$ altre arti. Atti del secondo Convegno nazionale (Bologna, 3-4 giugno 2010). Milano: Ledizioni, 2011. p. 81-112.

JEDLOWSKI, P. Storie comuni. La narrazione nella vita quotidiana. Milano: Bruno Mondadori 2000.

MAGRIS, C. Davanti alla legge. Trieste: EUT, 2006a. 
MAGRIS, C. Letteratura e Diritto. Davanti alla legge. Cuadernos de Filología Italiana, v. 13, p. 175-181, 2006b.

MARCHETTO, G.; CAU, M. (Eds.). Droit et littérature. Laboratoire italien: politique et société, n. 5, p. 7-12, 2004.

MARIANI MARINI, A.; BAMBI, F. (Eds.). Lingua e diritto. Scritto $e$ parlato nelle professioni legali. Pisa University Press, Pisa 2013.

MINDA, G. Postmodern Legal Movements. Law and Jurisprudence at Century's End, New York and London: New York U.P., 1995. Tr. it. Teorie Postmoderne del diritto. Bologna: Il Mulino, 2001.

MITTICA, M. P. Diritto e letteratura. Disciplina, metodologia o movimento? In: N. MARASCHIO, D. DE MARTINO, G. STANCHINA (Eds.). Lingue e diritti, I, Le parole della discriminazione. Diritto e letteratura. Firenze, 14 e 16 novembre 2013. Firenze: Accademia della Crusca, 2014. p. 111-138.

MITTICA, M. P. Diritto e Costruzione narrativa. La connessione tra diritto e letteratura. Spunti per una riflessione. Tigor, n. 1, p. 14-23, 2010.

MITTICA, M. P. Raccontando il possibile. Eschilo e le narrazioni giuridiche. Milano: Giuffré, 2006.

MORTARA GARAVELLI, B. Le parole e la giustizia. Divagazioni grammaticali e retoriche su testi giuridici italiani. Torino: Einaudi 2001.

NOUSSIA, M. (Ed.). Solone. Frammenti dell'opera poetica. Trad. M. Fantuzzi. Milano: BUR, 2001.

NUSSBAUM, M. C. Not For Profit: Why Democracy Needs the Humanities. Princeton: Princeton University Press, 2010. Tr. it. Non per profitto. Perché le democrazie hanno bisogno della cultura umanistica. Bologna: Il Mulino, 2011.

NUSSBAUM, M. C. Hiding from Humanity. Disgust, Shame, and the Law. Princeton: Princeton University Press, 2004. Tr. it. Nascondere l'umanità: il disgusto, la vergogna, la legge. Milano: Carocci, 2005.

NUSSBAUM, M. C. Upheavals of Thought: The Intelligence of Emotions. Cambridge: Cambridge University Press, 2001. Tr. it. L’intelligenza delle emozioni. Bologna: Il Mulino, 2009.

NUSSBAUM, M. C. Cultivating Humanity: A Classical Defense of Reform in Liberal Education. Cambridge (MA): Harvard University Press, 1997. Tr. it. Coltivare l'umanità, Roma: Carocci, 1999.

NUSSBAUM, M. C. Poetic Justice. The Literary Imagination and Public Life. Boston: Beacon Press, 1995. Tr. it. Il giudizio del poeta. Immaginazione letteraria e vita civile. Milano: Feltrinelli, 1996.

OLSON, G. De-Americanizing Law and Literature Narratives: Opening Up the Story. Law and Literature, v. 22, n. 2, p. 338-364, 2010.

OST, F. La justice, ses alternatives et ses symboles. Venger, pardonner ou juger? Variations littéraires. In : MASTOR, W. ; MINIATO, L. (Eds.). Les figures du procès au delà des frontières. Paris: Dalloz, 2014.

OST, F. Raconter la loi. Aux sources de l'imaginaire juridique. Paris: Odile Jacob, 2004. Tr. it. Mosè, Eschilo, Sofocle. Bologna: Il Mulino, 2007. 
OST, F.; VAN EYNDE, L.; GÉRARD, P.; VAN DE KERCHOVE, M. (Eds.). Lettres et lois. Le droit au miroir de la littérature. Bruxelles: Publications des Facultés Universitaires Saint-Louis, 2001.

PERGOLESI, F. Diritto e giustizia nella letteratura moderna narrativa e teatrale. Bologna: Zuffi, 1956.

PERGOLESI, F. Il diritto nella letteratura. Archivio Giuridico "Filippo Serafini”, v. XCVII, fasc. 1, p. 61-104, 1927.

PITTION, J.-P. Writing the law/Righting the law in Shakespeare's time. In: CARPI, D. (Ed.), Shakespeare and the Law. Ravenna: Longo 2003. p. 5969.

POZZO, B. (Ed.). Teaching Law through the Looking Glass of Literature. Berne: Stampfli, 2010.

POSNER, R. Law and Literature. 3. ed. Cambridge (MA)/ London: Harvard University Press, 2009.

QUAGLIONI, D. La Vergine e il diavolo. Letteratura e diritto, letteratura come diritto. Laboratoire italien, n. 5, p. 39-55, 2005.

RADBRUCH, G. Psicologia del sentimento giuridico dei popoli. Rivista internazionale di filosofia del diritto, v. XVIII, p. 241-251, 1938.

RESTIVO, G. Amleto e la giustizia. In: MITTICA, M. P. (Ed.). Diritto e narrazioni. Temi di letteratura, diritto e altre arti. Milano: Ledizioni, 2011.

SANSONE, A. Diritto e Letteratura. Milano: Giuffrè, 2001.

SARAT, A.; ANDERSON, M; FRANK, C. O. (Eds.). Law and the Humanities. An Introduction. Cambridge: Cambridge University Press, 2010.

SILBEY, S. S. Everyday Life and the Constitution of Legality. In: JACOBS, M. D.; HANRAHAN, N. (Eds.). The Blackwell Companion to the Sociology of Culture. Malden (MA): Blackwell, 2005. p. 332-345.

SMORTI, A. Narrazioni. Cultura, memoria e formazione del Sé. Milano: Bruno Mondadori 2007.

TUZET, G. Diritto e letteratura: Finzioni a confronto. Annali dell'Università di Ferrara, Sc. Giur., Nuova Serie, v. XIX, p. 179-204, 2005.

VESPAZIANI, A. Costituzione, Comparazione, Traduzione: Saggi di Diritto e Letteratura. Torino: Giappichelli, 2012.

VESPAZIANI, A. Cooperazione e traduzione: dalla letteratura al diritto. In: MITTICA, M. P. (Ed.). Diritto e narrazioni. Temi di diritto, letteratura e altre arti. Atti del secondo Convegno nazionale (Bologna, 3-4 giugno 2010). Milano: Ledizioni, 2011. p. 313-324.

VESPAZIANI, A. Per un'ermeneutica della metafora giuridica. In: RIDOLA, P. et al. Scritti in onore di A.A. Cervati. Roma: Aracne, 2009.

WEISBERG, R. Law-Literature Enterprise. Yale Journal of Law \& Humanities, v. 1, n. 1, p. 1-67, 1989. 
WHITE, J. B. The Cultural Background of "The Legal Imagination". In: SARAT, A.; FRANK, C.; ANDERSON, M. (Eds.). Teaching Law and Literature. New York: Modern Language Association, 2011.

WHITE, J. B. Law and Literature: "No Manifesto". Mercer Law Review, n. 39, p. 739-752, 1987-1988.

WHITE, J. B. When Words Lose Their Meaning: Constitutions and Reconstitutions of Language, Character, and Community. Chicago: University of Chicago Press, 1984. Tr. it. Quando le parole perdono il loro significato. Linguaggio, individuo, comunità. Milano: Giuffrè, 2010.

WHITE, J. B. The legal Imagination. Studies in the Nature of Legal Thought and Expression. Boston: Little Brown, 1973.

WIGMORE, J. A List of Legal Novels. Illinois Law Review, v. 2, p. 574-593, 1908.

Lingua originale: Italiano

Ricevuto: 15/02/2015

Accettato: 21/04/2015 